\title{
Genome Biology and the Evolution of Cell-Size Diversity
}

\author{
Rachel Lockridge Mueller \\ Department of Biology, Colorado State University, Fort Collins, Colorado 80523-1878 \\ Correspondence: rlm@colostate.edu
}

Cell size is highly variable among different species across the Tree of Life. For decades, biologists have generated hypotheses to explain this variation, in many cases, drawing on the correlations that exist among cell size, genome size, nucleus size, and various physiological and developmental parameters. In recent years, our understanding of the molecular processes that generate variation in genome size over evolutionary time, as well as the processes that maintain homeostasis in cell size over ontogenetic time, has increased dramatically. The goal of this article is to highlight how information from these fields can be integrated to generate new hypotheses to explain cell-size diversity.

Evolutionary biologists have been interested in Ethe diversity of genome sizes among species for decades. Across the Tree of Life, the size of the genome is correlated with many other features, for example, the size of the nucleus, the size of the cell, the rate of metabolism, and the rate of development (Gregory 2005a,b). Much of the previous work aimed at understanding the evolutionary forces shaping genome size diversity focused on these correlations, generating hypotheses to explain genome size diversity in light of their existence (Gregory 2001). In the broadest sense, these hypotheses shared the common goal of determining which evolutionary forcemutation, selection, or drift-acted as the primary driver of genome-/cell-size evolution. More specifically, this earlier work attempted to pinpoint (1) which, if any, of these correlated traits were direct targets of natural selection, and (2) what might underlie these multitrait correlations. These hypotheses were notoriously difficult to test, reflecting, in part, a lack of understanding of the molecular mechanisms regulating genome size, nucleus size, and cell size.

Since this initial work, however, our knowledge of such molecular mechanisms has increased dramatically. This new information presents an opportunity to revisit these, now, classic hypotheses for the evolution of genome-/cellsize diversity, updating them to spur novel data collection and analyses. The goal of this review is to focus on how specific advances in our understanding of the molecular processes (1) generating variation in genome size, (2) regulating cell size, and (3) regulating nucleus size can reinvigorate the debate surrounding these central questions about the evolution of the cell.

What regulates the size of a cell? Cell size is variable among tissue types within single organisms. In addition, cell size is variable

Editors: Rebecca Heald, Iswar K. Hariharan, and David B. Wake

Additional Perspectives on Size Control in Biology: From Organelles to Organisms available at www.cshperspectives.org

Copyright (C) 2015 Cold Spring Harbor Laboratory Press; all rights reserved; doi: 10.1101/cshperspect.a019125

Cite this article as Cold Spring Harb Perspect Biol 2015;7:a019125 
throughout ontogeny. However, within tissue types or developmental stages, cell size is remarkably constant; cell-to-cell size variation is minimal. How is such constancy achieved within organisms? At the same time, how has such diversity in cell size evolved across the Tree of Life? The former question has been the topic of much recent interest, with work in yeast and mammalian systems providing critical insights into some of the mechanisms determining cell size (Marshall et al. 2012; Kafri et al. 2013; Lloyd 2013). The latter question has received much less attention, but recent advances in several fields suggest that the time may be ripe for successful interdisciplinary approaches to studying cell-size evolution.

The question of what regulates cell size can be decomposed into several composite parts: (1) What dictates the "target" size of a cell? (2) How do cells sense whether they are at, over, or under this target size? (3) How do cells return to this target size if caused to deviate from it? Evolutionary changes in cell size could, at least in theory, reflect changes to any of these processes, reflecting associated evolutionary changes to different underlying genetic pathways. For example, the target size of a cell could evolve to a new set point, but the underlying mechanisms for sensing and correcting cell size (i.e., maintaining homeostasis) could remain unchanged. Conversely, at least in principle, the target size of a cell could remain unchanged, but cellular ability to sense or correct cell size could evolve, shifting actual cell size away from the target size in a consistent way. Here, I briefly review what is known about the setting, sensing, and maintenance of cell size and highlight potentially fruitful areas for evolutionary analysis.

What dictates the target size of a cell in a given species? This is likely the most relevant question for understanding the evolution of cell size; however, it is also the most difficult to translate into testable hypotheses. Because cell size is correlated with a number of other features-for example, physiological traits, including metabolic rate, life history traits, including developmental rate-evolutionary biologists have posed a number of hypotheses in which selection acting on one of these correlates push- es cell size toward an optimum value (i.e., a target size) that maximizes organismal fitness (Gregory 2005a). In vertebrates, for example, these hypotheses include selection for high metabolic rate (and, thus, small cell size) in birds and bats, as well as low metabolic rate (and, thus, a "frugal" metabolic strategy and large cells) in salamanders (Olmo and Morescalchi 1975; Szarski 1983; Burton et al. 1989; Cavalier-Smith 1991; Hughes and Hughes 1995; Waltari and Edwards 2002). Additional hypotheses include selection for rapid developmental rate (and, thus, small cell size) in both vertebrate and invertebrate taxa with complex developmental programs (Gregory 2002). Taken together, these hypotheses have in common a primary role for natural selection acting on a correlate of cell size in dictating the target size of a cell. Although such hypotheses may focus on the fitness consequences of the size of one particular cell type (e.g., red blood cells), the variation in size among cell types within organisms is typically not explicitly addressed. Thus, selection is inferred to act on some subset of the total types of cells in a multicellular organism, with the sizes of others being indirectly affected.

Cell size is also correlated with subcellular traits, including the size of the genome; thus, evolutionary biologists have also posed hypotheses for what dictates the target size of a cell that focus on the genome-/cell-size correlation. However, these hypotheses are fundamentally different from those that invoke selection targeting a cell-size correlate that impacts organismal fitness. For example, in vertebrates, large cells in salamanders and lungfishes have been hypothesized to result from exceptionally high levels of transposable element (TE) accumulation in the genome (Roth et al. 1994); detailed analyses of such TE sequences have begun to be undertaken in recent years (Metcalfe et al. 2012; Sun et al. 2012). This genomic expansion, in turn, dictates an increase in cell size. In this evolutionary scenario, target cell size is not optimized by selection, but rather is an emergent property of genome-level processes (Roth et al. 1994). Selection to maintain smaller cell size is inferred to be either too weak (i.e., differences in fitness among individuals with genomes of dif- 
ferent sizes are minimal) or too inefficient (i.e., genetic drift is too strong, relative to fitness differences among individuals with genomes of different sizes) to counteract the expansion of both genome and cell sizes (Lynch 2007). The mechanisms by which genome expansion via TE accumulation translates into an increase in cell size were not explicitly considered in these hypotheses and, in fact, remain unknown. Changes to other traits correlated with cell size, for example, metabolic rate, developmental rate, and morphological complexity, were also inferred to follow from changes in genome size, but not be direct targets of natural selection themselves (Roth et al. 1990, 1997). Much of the support for these nonadaptive hypotheses of target cell-size determination, at least in vertebrates, has come from analyses of morphological data.

Underlying both of these classes of hypotheses, those that see a primary role for selection in shaping target cell size, and those that see target cell size emerging from nonadaptive (at the organismal level) intragenomic dynamics, is an implied mechanism coupling genome and cell size. Thus, if selection shapes target cell size, then genome size changes in a correlated fashion. Conversely, if genome size evolves, target cell size changes in a correlated fashion. Although such a mechanism remains unknown, new data have suggested new models for the connection between genome and cell size. In addition, our understanding of the molecular processes generating variation in genome size has increased dramatically over the past decade. Given all of this new information, it is becoming possible to refine hypotheses about how target cell size evolves to address more explicitly (1) the mechanism coupling genome and cell size, and (2) the role for natural selection in shaping the evolution of these two correlated traits.

Genome size changes over evolutionary time by the addition or subtraction of different types of sequences through different processes. Increases in ploidy, either cell specific (e.g., endoreplication, in which specific cells within a developing organism undergo DNA replication without associated cell division) or organism wide (e.g., polyploidization), are correlated with increases in cell size (Lee et al. 2009; Marshall et al. 2012). The mechanisms underlying this correlation remain unclear, but hypotheses include (1) doubling (or further increasing) transcriptional output results in doubling (or further increasing) cell size through its impact on overall rates of anabolic and catabolic pathways (Lloyd 2013), or (2) doubling (or further increasing) of the transcript levels of a particular inhibitory regulator of cell cycle initiation means that the threshold concentration will be met in a proportionally larger cell (Marshall et al. 2012). These are viable hypotheses for endoreplication because genes, and the networks in which they are embedded, are still expected to encode functional products. It may be a viable hypothesis for evolutionarily extremely recent polyploids as well, in which paralog loss has not yet occurred. However, it is not a viable hypothesis for older polyploids, in which paralog loss-of-function, subfunctionalization, and neofunctionalization will have eliminated the initial increase in transcriptional/translational output (Force et al. 1999; Soria et al. 2014); such species still show a relationship between genome and cell size, which must reflect other mechanisms. In addition, variation in TE levels is one of the primary generators of genome size diversity across taxa. Such diversity is correlated with cell size, but would not be expected to reflect any differences in expression levels of endogenous genes, again suggesting other mechanisms. What might these mechanisms be?

Cell size within populations of dividing cells reflects the coordinated interaction between cell growth and cell division (Jorgensen et al. 2002); division following less cell growth produces smaller cells, whereas division following more cell growth produces larger cells (Edgar and Kim 2009). The mechanisms underlying the interaction between these two processes are the subject of intense research (Ferrezuelo et al. 2012; Hoose et al. 2012; Kafri et al. 2013; Moretto et al. 2013). Two general classes of models have been put forward to explain how cells sense the correct time at which to divide: "timer" models and "sizer" models (Turner et al. 2012). In the timer models, cells keep track of the duration of time that has elapsed since the last cell division. 
Assuming linear cell growth, this yields a constant cell size at which division is initiated. Across taxa, evolved differences in the amount of time allowed to elapse among cell division events would translate into differences in cell size. In the sizer models, cells keep track of their actual size. This is necessary to maintain constant cell size in populations of cells that grow exponentially, or that grow through combined periods of exponential and linear growth. Across taxa, evolved differences in the size at which cell division is initiated would translate into differences in cell size. Sizer models encompass a variety of proposed mechanisms; for example, in the rod-shaped yeast Schizosaccharomyces pombe, evidence suggests that the sizer mechanism detects cell length (Martin and Berthelot-Grosjean 2009; Moseley et al. 2009). More generally, however, sizer models include a protein-synthesisrate sensor as an indicator of size. As cell size increases, ribosome content increases, and protein synthesis rate increases. Although protein concentration per unit of cytoplasm remains constant, overall protein levels are higher in a larger cell. Thus, sizer models typically hypothesize that protein levels are calibrated against levels of another substance that does not become more abundant with increasing cell size (Turner et al. 2012).

DNA was first proposed as such a "standard" in the 1960s, although empirical support for this model has been lacking (Donachie 1968; Fantes et al. 1975; Marshall et al. 2012). Recently, however, new data suggests that this model may hold in the budding yeast Saccharomyces cerevisiae. More specifically, Wang et al. (2009) propose that the $G_{1}$ cyclin protein $C \ln 3$ binds to the numerous SBF-binding-site sequences in the genome, offering a measure of $\mathrm{Cln} 3$ levels calibrated against levels of genomic DNA. Once a threshold ratio of $\mathrm{Cln} 3 / \mathrm{SBF}$ binding site is reached, entry into the cell cycle occurs. The extent to which this, or similar, mechanisms underlie cell-size control in other taxa remains unknown. However, across eukaryotes, the topology of the molecular networks regulating entry into the cell cycle (i.e., $\mathrm{G}_{1}-\mathrm{S}$ transition), as well as the networks' dynamic properties, are conserved, despite seemingly unrelated amino acid sequences of the proteins performing specific roles within the networks (Cross et al. 2011). Thus, a potential sizer model that calibrates cytoplasmic protein levels against available DNA binding sites presents an interesting model for the tight correlation between genome and cell size across the Tree of Life worthy of continued investigation. Because the correlation between genome and cell size also includes a correlation with the size of the nucleus, older hypotheses for this multitrait correlation also included an active role for nucleus size in the proposed mechanisms, linking all three traits. However, recent data suggests that the size of the nucleus responds directly to cytoplasmic volume, irrespective of the amount of DNA (Edens et al. 2013); a direct connection between genome and cell size is consistent with this model of nuclear size determination.

Given the diversity of sequences that underlie variation in genome size across taxa, the nature of the protein/DNA binding interaction would have to be quite variable. For example, in cases, in which genome expansion reflects increases in ploidy, or even duplications of partial chromosomal segments, existing binding sites are duplicated. In contrast, in cases, in which genome expansion reflects increased TE load, putative binding sites that increase in availability throughout the genome would have to be TE derived, and the sequence diversity encompassed by the classes of TEs populating eukaryotic genomes translates into many diverse potential binding sites (Wicker et al. 2007). Although TE promoters are known to have played key roles in the evolution of gene regulatory networks (Feschotte 2008), TE's potential roles in calibrating protein-binding-based cell-size monitoring remain unexplored.

If cells sense their target size using an interaction between cytoplasmic volume and DNA content, then the DNA sequences underlying genome size variation would present an opportunity for assessing the role for natural selection in shaping the evolution of these two correlated traits. Variation in genome size is produced by heritable insertion and deletion mutations (indels) in the germline. Two specific types of indels have been hypothesized as major drivers of 
genome size variation: (1) insertions of new TE copies by transposition, and (2) deletions of existing TE copies by ectopic recombination (i.e., nonallelic homologous recombination). TE transposition occurs because of a failure of the cell's TE-silencing machinery. Ectopic recombination occurs because of an error in the identification of the template to be used for double-strand DNA break repair during meiotic and/or germline mitotic recombination (Bennetzen and Kellogg 1997; Tian et al. 2009). Natural variation in both TE activity levels and ectopic recombination levels exists across taxa (Furano et al. 2004; Frahry et al. 2015), suggesting differences in function of the proteins regulating transposition and recombination. The molecular mechanisms of such protein functions are the topic of intense research (MineHattab and Rothstein 2012; Dion and Gasser 2013; Clark and Lau 2014).

My goal is to use the cell's TE-silencing machinery as an example for outlining predicted patterns of protein sequence evolution under two evolutionary scenarios: (1) selection pushing cell size toward an optimum value (i.e., a target size) that maximizes organismal fitness (Rayburn et al. 1994; Cavalier-Smith 2005), and (2) target cell size emerging from nonadaptive (at the organismal level) intragenomic dynamics (Roth et al. 1994). Differences in TE activity and abundance exist across species, in many cases correlated with differences in overall genome and cell size. However, to date, a role for variation in the cell's TE-silencing machinery in generating such differences remains largely unexplored (Arensburger et al. 2011). Furthermore, the evolutionary forces (selection, drift, mutation) that may have acted to generate any variation in the cell's TE-silencing machinery remain unexplored.

TE proliferation is mutagenic; novel insertions can impact protein structure, gene expression, and the frequency of chromosomal rearrangement (Montgomery et al. 1987; Petrov et al. 2003; De Gobbi et al. 2006). Eukaryotic cells suppress TE proliferation, although it is never completely silenced-some level of activity, variable across species, persists. Novel TE insertions are now known to be suppressed by several pathways involving small RNA molecules. Differences in these pathways are apparent among different model organisms, both within and among major multicellular eukaryotic clades (i.e., animals, plants, and fungi) (Dumesic and Madhani 2014). Here, I will focus on the Piwi-interacting RNA ( piRNA) pathway, which acts as a "genome defense" system that, for example, limits TE activity in the animal germline (Siomi et al. 2011). Many aspects of this pathway, as well as analogous pathways in other organisms, have recently been reviewed elsewhere (e.g., Siomi et al. 2011; Guzzardo et al. 2013; Luteijn and Ketting 2013; Saito 2013; Sato and Siomi 2013); thus, only a very brief overview is provided here.

TE suppression in the animal germline by the piRNA pathway requires piRNA molecules complementary to TE sequences. Mature piRNAs are typically $24-31 \mathrm{nt}$ in length, and many have a $U$ in the $5^{\prime}$ position. Mature piRNAs active in TE suppression are produced through the processing of long precursor transcripts of genomic loci that contain active TEs or remnants of TE insertions. piRNAs in both sense and antisense orientations relative to TE transcripts contribute to TE suppression. Antisense piRNAs, complexed with effector proteins in the Piwi clade of the Argonaute family (Piwi proteins hereafter), target TE sequences for transcriptional and posttranscriptional suppression (Senti and Brennecke 2010). Transcriptional suppression results from chromatin modification to epigenetically silence TE loci, and posttranscriptional suppression results from destruction of TE transcripts (Hammond et al. 2001; Brennecke et al. 2008; Malone and Hannon 2009; Law and Jacobsen 2010; Siomi et al. 2011). Sense piRNAs contribute to the continued production of antisense piRNAs (Brennecke et al. 2007; Gunawardane et al. 2007). Thus, piRNA-mediated TE suppression requires that (1) mature piRNAs are produced in the germline that match the active TE population in the genome (Kelleher and Barbash 2013), (2) the TE-targeting piRNAs are bound effectively by Piwi proteins, and (3) all of the Piwi pathway proteins that contribute to TE silencing (e.g., through target recognition or ef- 
fector function) are functional, either alone and/or as part of their relevant functional complexes. There are many ways in which variation in such TE suppression across species could lead to differences in TE content and, by extension, genome and cell size. Here, I outline several possibilities and discuss how they could be explored to assess the role for natural selection in shaping the evolution of genome and cell size.

First, species could vary in the degree of "match" between the pool of mature piRNA molecules in their germline and the active TE population in their genome. The strength of selection for TE suppression is inferred, based on population genetic models, to be strongest for TE families with the most deleterious fitness consequences (i.e., very high activity levels). However, additional fitness increases associated with incrementally improving existing repression are inferred to be small. Based on this, piRNA pools are predicted to target all active TEs, although targeting effectiveness may not scale with TE activity level (Charlesworth and Langley 1986; Nuzhdin 1999; Blumenstiel 2011; Kelleher and Barbash 2013). Across species, diversity in (1) the number of active TE families that lack representation in the piRNA pool, and (2) the abundance of piRNAs targeting active families could both contribute to variation in TE load. More specifically, the piRNA pools of species with larger genomes would be predicted to have more untargeted TE families and fewer piRNA molecules per active TE locus. This prediction is consistent with genomic expansion via TE accumulation reflecting less efficient piRNA-mediated TE silencing.

Second, species could vary in the binding affinity of Piwi proteins to TE-targeting piRNAs. piRNAs show extraordinary sequence diversity (Vourekas et al. 2012; Cora et al. 2014); thus, Piwi proteins must bind piRNAs that encompass a huge range of nucleotide sequences. However, despite this requirement for binding diverse sequences, Piwi proteins do show binding preference based on the $5^{\prime}$ nucleotide and the sense/antisense orientation of the piRNA with respect to target TEs (Cora et al. 2014). Furthermore, other Argonaute proteins show differential binding affinities within other classes of small RNAs (e.g., microRNAs [miRNAs]) (Zhang et al. 2014). Thus, across species, variation in the binding affinity of Piwi proteins for diverse sequences could contribute to variation in TE load. More explicitly, Piwi proteins of species with larger genomes comprised of diverse TEs would be predicted to bind a less diverse set of piRNA sequences with high affinity. As a consequence, piRNAs targeting some TEs might not be bound by Piwi proteins with high affinity, reducing TE suppression. This prediction is consistent with genomic expansion via TE accumulation reflecting less efficient piRNA-mediated TE silencing.

Finally, species could vary in diverse ways related to the efficiency of both target recognition by the piRNA/Piwi protein complex, as well as effector function (i.e., transcriptional and/or posttranscriptional silencing). More specifically, target recognition and effector function in species with larger genomes would be predicted to be less efficient. This prediction is consistent with genomic expansion via TE accumulation reflecting less efficient piRNAmediated TE silencing.

How could these possibilities be explored to assess the role for natural selection in shaping the evolution of genome and cell size? To date, data appropriate for comparing piRNA-mediated TE suppression among species with different TE loads and genome sizes are scarce, and few analyses along these lines have been performed (Arensburger et al. 2011). The first step would be to identify a clade of species that shows both stasis and substantial evolutionary changes in genome size. The second step would be to examine, for each species, (1) the match between the pool of mature piRNAs in the germline and the active TE population in the genome, (2) the binding affinity of Piwi proteins for diverse TE-targeting piRNAs, and (3) the efficiency of both target recognition by the piRNA/Piwi protein complex, as well as effector function. The third step would be to test whether evolutionary changes in any of these aspects of piRNA-mediated TE suppression coincide with evolutionary changes in genome size. Aspects of the piRNA pathway that change with genome size would be candidates for producing variation in TE abun- 
dance/genome size across species. Once identified, the fourth step would be to test the sequences encoding the proteins underlying such candidates for evidence of natural selection. Assuming the target species do not encompass huge evolutionary distances, this could be performed using standard amino-acid-divergencebased tests for selection based on the ratio of (1) nonsynonymous to synonymous substitutions $(d N / d S)$, or (2) radical to conservative amino acid replacements $(K r / K c) . d N / d S$ and $K r / K c$ values are used to identify the primary evolutionary forces shaping changes in amino acid sequences (Hanada et al. 2007); for example, $d N / d S$ values $=1$ indicate sequences evolving under neutral processes (i.e., genetic drift), whereas $d N / d S$ values $>1$ indicate sequences evolving under positive natural selection (i.e., by adaptive evolution). Under a scenario in which cell size reflects selection toward a target size that maximizes organismal fitness, piRNA pathway proteins in lineages that have experienced dramatic increases in genome size should show amino acid substitution patterns consistent with positive natural selection/adaptive evolution. In contrast, under a scenario in which target cell size emerges from a genome size that reflects genome-level processes (i.e., TE proliferation with minimal impacts on organismal fitness), piRNA pathway proteins in lineages that have experienced dramatic increases in genome size should show amino acid substitution patterns consistent with nonadaptive processes / neutral evolution.

To date, much about the piRNA pathway proteins that silence TEs remains incompletely understood (Clark and Lau 2014). This includes: (1) the mechanisms by which the appropriate precursor transcripts (including TEderived transcripts) are selected for processing into mature piRNAs, which underlies the match between piRNAs and active TEs; (2) the mechanisms by which piRNAs are sorted, loaded onto, and bound by appropriate Piwi proteins, which underlies the binding affinity of Piwi proteins to diverse TE-targeting piRNAs; and (3) many aspects of target recognition and effector function. However, our understanding of piRNA biology continues to improve, and these proteins could emerge as an excellent system for assessing the role for natural selection in shaping the evolution of genome and cell size.

\section{CONCLUDING REMARKS}

The evolutionary forces shaping the diversity of cell sizes across the Tree of Life remain largely unknown. Furthermore, the coordinated evolution of genome and cell size across the Tree of Life, despite major differences in genome content and cell architecture, remains largely unexplained. Here, I presented several examples of how information about the molecular processes that regulate cell size, as well as maintain genome size and integrity, can be leveraged to generate testable hypotheses about cell-size evolution. There are many potentially fruitful avenues of evolutionary research that could leverage other information about the processes regulating cell-size homeostasis (e.g., the balance between anabolic and catabolic pathways, the relationship between mitochondrial metabolism and lipid biosynthesis, the maintenance of osmotic balance across cell membranes) to generate testable hypotheses (Koivusalo et al. 2009; Lloyd 2013; Miettinen et al. 2014). Given all of these opportunities, the future looks bright for evolutionary cell biology.

\section{ACKNOWLEDGMENTS}

I thank T. Montgomery, D. Sloan, and members of the Sloan and Mueller laboratories for helpful comments and discussion. Research in the Mueller laboratory is supported by the National Science Foundation (NSF-DEB 1021489).

\section{REFERENCES}

Arensburger P, Hice RH, Wright JA, Craig NL, Atkinson PW. 2011. The mosquito Aedes aegypti has a large genome size and high transposable element load but contains a low proportion of transposon-specific piRNAs. BMC Genomics 12: 606

Bennetzen JL, Kellogg EA. 1997. Do plants have a one-way ticket to genomic obesity? Plant Cell 9: 1509.

Blumenstiel JP. 2011. Evolutionary dynamics of transposable elements in a small RNA world. Trends Genet 27: 23-31. 
R.L. Mueller

Brennecke J, Aravin AA, Stark A, Dus M, Kellis M, Sachidanandam R, Hannon GJ. 2007. Discrete small RNA-generating loci as master regulators of transposon activity in Drosophila. Cell 128: 1089-1103.

Brennecke J, Malone CD, Aravin AA, Sachidanandam R, Stark A, Hannon GJ. 2008. An epigenetic role for maternally inherited piRNAs in transposon silencing. Science 322: 1387.

Burton DW, Bickham JW, Genoways HH. 1989. Flow-cytometric analyses of nuclear DNA content in four families of neotropical bats. Evolution 43: 756-765.

Cavalier-Smith T. 1991. Coevolution of vertebrate genome, cell, and nuclear sizes. In Symposium on the evolution of terrestrial vertebrates selected symposia and monographs UZI (ed. Ghiara G, Angelini F, Olmo E, Varano L), pp. 51-86. Mucchi, Modena, Italy.

Cavalier-Smith T. 2005. Economy, speed and size matter: Evolutionary forces driving nuclear genome miniaturization and expansion. Ann Bot 95: 147-175.

Charlesworth B, Langley CH. 1986. The evolution of selfregulated transposition of transposable elements. Genetics 112: 359-383.

Clark JP, Lau NC. 2014. Piwi proteins and piRNAs step onto the systems biology stage. In Systems biology of RNA binding proteins (ed. Yeo GW), pp. 159-197. Springer, New York.

Cora E, Pandey RR, Xiol J, Taylor J, Sachidanandam R, McCarthy AA, Pillai RS. 2014. The MID-PIWI module of Piwi proteins specifies nucleotide and strand biases of piRNAs. RNA 20: 773-781.

Cross FR, Buchler NE, Skotheim JM. 2011. Evolution of networks and sequences in eukaryotic cell cycle control. Philos Trans R Soc Lond B Biol Sci 366: 3532-3544.

De Gobbi M, Viprakasit V, Hughes JR, Fisher C, Buckle VJ, Ayyub H, Gibbons RJ, Vernimmen D, Yoshinaga Y, de Jong PJ, et al. 2006. A regulatory SNP causes a human genetic disease by creating a new transcriptional promoter. Science 26: 1215-1217.

Dion V, Gasser SM. 2013. Chromatin movement in the maintenance of genome stability. Cell 152: 1355-1364.

Donachie WD. 1968. Relationship between cell size and time of initiation of DNA replication. Nature 219: 1077-1079.

Dumesic PA, Madhani HD. 2014. Recognizing the enemy within: Licensing RNA-guided genome defense. Trends Biochem Sci 39: 25-34.

Edens LJ, White KH, Jevtic P, Li X, Levy DL. 2013. Nuclear size regulation: From single cells to development and disease. Trends Cell Biol 23: 151-159.

Edgar BA, Kim KJ. 2009. Sizing up the cell. Science 325: $158-159$.

Fantes PA, Grant WD, Pritchard RH, Sudbery PE, Wheals AE. 1975. The regulation of cell size and the control of mitosis. J Theor Biol 50: 213-244.

Ferrezuelo F, Colomina N, Palmisano A, Garí E, Gallego C, Csikász-Nagy A, Aldea M. 2012. The critical size is set at a single-cell level by growth rate to attain homeostasis and adaptation. Nat Commun 3: 1012.

Feschotte C. 2008. Transposable elements and the evolution of regulatory networks. Nat Rev Gen 9: 397-405.

Force A, Lynch M, Pickett FB, Amores A, Yan YL, Postlethwait J. 1999. Preservation of duplicate genes by com- plementary, degenerative mutations. Genetics 151: 15311545.

Frahry MB, Sun C, Chong R, Mueller RL. 2015. Low levels of LTR retrotransposon deletion by ectopic recombination in the gigantic genomes of salamanders. J Mol Evol 80: $120-129$.

Furano AV, Duvernell DD, Boissinot S. 2004. L1 (LINE-1) retrotransposon diversity differs dramatically between mammals and fish. Trends Genet 20: 9-14.

Gregory TR. 2001. Coincidence, coevolution, or causation? DNA content, cell size, and the C-value enigma. Biol Rev Camb Philos Soc 76: 65-101.

Gregory TR. 2002. Genome size and developmental complexity. Genetica 115: 131-146.

Gregory TR. 2005a. The C-value enigma in plants and animals: A review of parallels and an appeal for partnership. Ann Bot 95: 133-146.

Gregory TR. 2005b. The evolution of the genome. Academic, New York.

Gunawardane LS, Saito K, Nishika KM, Kawamura Y, Nagami T, Siomi H, Siomi MC. 2007. A slicer-mediated mechanism for repeat-associated siRNA $5^{\prime}$ end formation in Drosophila. Science 315: 1587-1590.

Guzzardo PM, Muerdter F, Hannon GJ. 2013. The piRNA pathway in flies: Highlights and future directions. Curr Opin Genet Dev 23: 44-52.

Hammond SM, Boettcher S, Caudy AA, Kobayashi R, Hannon GJ. 2001. Argonaute2, a link between genetic and biochemical analyses of RNAi. Science 293: 1146.

Hanada K, Shiu S-H, Li W-H. 2007. The nonsynonymous/ synonymous substitution rate ratio versus the radical/ conservative replacement rate ratio in the evolution of mammalian genes. Mol Biol Evol 24: 2235-2241.

Hoose SA, Rawlings JA, Kelly MM, Leitch MC, Ababneh QO, Robles JP, Taylor D, Hoover EM, Hailu B, McEnery KA. 2012. A systematic analysis of cell cycle regulators in yeast reveals that most factors act independently of cell size to control initiation of division. PLoS Genet 8: e1002590.

Hughes AL, Hughes MK. 1995. Small genomes for better flyers. Nature 377: 391.

Jorgensen P, Nishikawa JL, Breitkreutz B, Tyers M. 2002. Systematic identification of pathways that couple cell growth and division in yeast. Science 297: 395-400.

Kafri R, Levy J, Ginzberg MB, Oh S, Lahav G, Kirschner MW. 2013. Dynamics extracted from fixed cells reveal feedback linking cell growth to cell cycle. Nature 494: 480-483.

Kelleher ES, Barbash DA. 2013. Analysis of piRNA-mediated silencing of active TEs in Drosophila melanogaster suggests limits on the evolution of host genome defense. Mol Biol Evol 30: 1816-1829.

Koivusalo M, Kapus A, Grinstein S. 2009. Sensors, transducers, and effectors that regulate cell size and shape. $J$ Biol Chem 284: 6595-6599.

Law JA, Jacobsen SE. 2010. Establishing, maintaining and modifying DNA methylation patterns in plants and animals. Nat Rev Gen 11: 204.

Lee HO, Davidson JM, Duronio RJ. 2009. Endoreplication: Polyploidy with purpose. Genes Dev 23: 2461-2477. 
Lloyd AC. 2013. The regulation of cell size. Cell 154: $1194-$ 1205.

Luteijn M, Ketting RF. 2013. PIWI-interacting RNAs: From generation to transgenerational epigenetics. Nat Rev Gen 14: $523-534$.

Lynch M. 2007. The origins of genome architecture. Sinauer, Sunderland, MA.

Malone CD, Hannon GJ. 2009. Small RNAs as guardians of the genome. Cell 136: 656-668.

Marshall WF, Young KD, Swaffer M, Wood E, Nurse P, Kimura A, Frankel J, Wallingford J, Walbot V, Qu X. 2012. What determines cell size? BMC Biol 10: 101.

Martin SG, Berthelot-Grosjean M. 2009. Polar gradients of the DYRK-family kinase Pom 1 couple cell length with the cell cycle. Nature 459: 852-856.

Metcalfe CJ, Filee J, Germon I, Joss J, Casane D. 2012. Evolution of the Australian lungfish (Neoceratodus forsteri) genome: A major role for CR1 and L2 LINE elements. Mol Biol Evol 29: 3529-3539.

Miettinen Teemu P, Pessa Heli KJ, Caldez Matias J, Fuhrer T, Diril MK, Sauer U, Kaldis P, Björklund M. 2014. Identification of transcriptional and metabolic programs related to mammalian cell size. Curr Biol 24: 598-608.

Mine-Hattab J, Rothstein R. 2012. Increased chromosome mobility facilitates homology search during recombination. Nat Cell Biol 14: 510-517.

Montgomery EA, Charlesworth B, Langley CH. 1987. A test for the role of natural selection in the stabilization of transposable element copy number in a population of Drosophila melanogaster. Genet Res 49: 31-41.

Moretto F, Sagot I, Daignan-Fornier B, Pinson B. 2013. A pharmaco-epistasis strategy reveals a new cell size controlling pathway in yeast. Mol Syst Biol 9: 707.

Moseley JB, Mayeux A, Paoletti A, Nurse P. 2009. A spatial gradient coordinates cell size and mitotic entry in fission yeast. Nature 459: 857-860.

Nuzhdin SV. 1999. Sure facts, speculations, and open questions about the evolution of transposable element copy number. Genetica 107: 129-137.

Olmo E, Morescalchi A. 1975. Evolution of the genome and cell sizes in salamanders. Experientia 31: 804-806.

Petrov DA, Aminetzach YT, Davis J, Bensasson D, Hirsch AE. 2003. Size matters: Non-LTR retrotransposable elements and ectopic recombination in Drosophila. Mol Biol Evol 20: $880-892$.

Rayburn AL, Dudley JW, Biradar DP. 1994. Selection for early flowering results in simultaneous selection for reduced nuclear DNA content in maize. Plant Breed 112: $318-322$.

Roth G, Rottluff B, Grunwald W, Hanken J, Linke R. 1990. Miniaturization in plethodontid salamanders (Caudata: Plethodontidae) and its consequences for the brain and visual system. Biol J Linn Soc 40: 165-190.
Roth G, Blanke J, Wake DB. 1994. Cell size predicts morphological complexity in the brains of frogs and salamanders. Proc Natl Acad Sci 91: 4796-4800.

Roth G, Nishikawa KC, Wake DB. 1997. Genome size, secondary simplification, and the evolution of the brain in salamanders. Brain Behav Evol 50: 50-59.

Saito K. 2013. The epigenetic regulation of transposable elements by PIWI-interacting RNAs in Drosophila. Genes Genet Syst 88: 9-17.

Sato K, Siomi MC. 2013. Piwi-interacting RNAs: Biological functions and biogenesis. Essays Biochem 54: 39-52.

Senti K-A, Brennecke J. 2010. The piRNA pathway: A fly's perspective on the guardian of the genome. Trends Genet 26: $499-509$.

Siomi MC, Sato K, Pezic D, Aravin AA. 2011. PIWI-interacting small RNAs: The vanguard of genome defence. Nat Rev Mol Cell Biol 12: 246-258.

Soria PS, McGary KL, Rokas A. 2014. Functional divergence for every paralog. Mol Biol Evol 31: 984-992.

Sun C, Shepard DB, Chong RA, Arriaza JL, Hall K, Castoe TA, Feschotte C, Pollock DD, Mueller RL. 2012. LTR retrotransposons contribute to genomic gigantism in plethodontid salamanders. Gen Biol Evol 4: 168-183.

Szarski H. 1983. Cell size and the concept of wasteful and frugal evolutionary strategies. J Theor Biol 105: 201-209.

Tian Z, Rizzon C, Du J, Zhu L, Bennetzen JL, Jackson SA, Gaut BS, Ma J. 2009. Do genetic recombination and gene density shape the pattern of DNA elimination in rice long terminal repeat retrotransposons? Genome Res 19: 2221.

Turner JJ, Ewald JC, Skotheim JM. 2012. Cell size control in yeast. Curr Biol 22: R350-R359.

Vourekas A, Zheng Q, Alexiou P, Maragkakis M, Kirino Y, Gregory BD, Mourelatos Z. 2012. Mili and Miwi target RNA repertoire reveals piRNA biogenesis and function of Miwi in spermiogenesis. Nat Struct Mol Biol 19: 773781.

Waltari E, Edwards SV. 2002. Evolutionary dynamics of intron size, genome size, and physiological correlates in archosaurs. Am Nat 160: 539-552.

Wang H, Carey LB, Cai Y, Wijnen H, Futcher B. 2009. Recruitment of $\mathrm{Cln} 3$ cyclin to promoters controls cell cycle entry via histone deacetylase and other targets. PLoS Biol 7: e1000189.

Wicker T, Sabot F, Hua-Van A, Bennetzen JL, Capy P, Chalhoub B, Flavell A, Leroy P, Morgante M, Panaud O. 2007. A unified classification system for eukaryotic transposable elements. Nat Rev Gen 8: 973-982.

Zhang X, Niu D, Carbonell A, Wang A, Lee A, Tun V, Wang Z, Carrington JC, Chang CE, Jin H. 2014. ARGONAUTE PIWI domain and microRNA duplex structure regulate small RNA sorting in Arabidopsis. Nat Commun 5: 5468. 


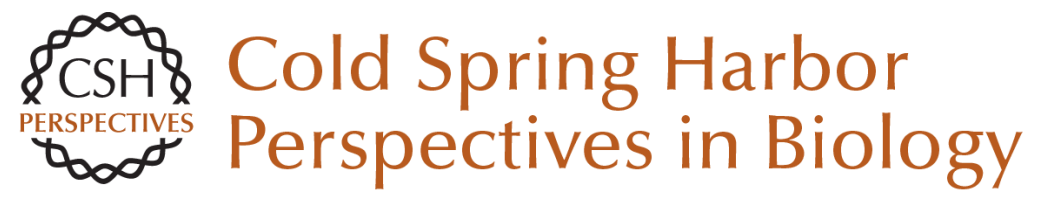

\section{Genome Biology and the Evolution of Cell-Size Diversity}

Rachel Lockridge Mueller

Cold Spring Harb Perspect Biol 2015; doi: 10.1101/cshperspect.a019125 originally published online August 7, 2015

\section{Subject Collection Size Control in Biology: From Organelles to Organisms}

Cell-Size Control

Amanda A. Amodeo and Jan M. Skotheim

Indeterminate Growth: Could It Represent the

Ancestral Condition?

Iswar K. Hariharan, David B. Wake and Marvalee H. Wake

The Systemic Control of Growth Laura Boulan, Marco Milán and Pierre Léopold

Genome Biology and the Evolution of Cell-Size Diversity

Rachel Lockridge Mueller

Size Scaling of Microtubule Assemblies in Early Xenopus Embryos

Timothy J. Mitchison, Keisuke Ishihara, Phuong Nguyen, et al.

The Influence of Genome and Cell Size on Brain Morphology in Amphibians Gerhard Roth and Wolfgang Walkowiak

The Opposing Actions of Target of Rapamycin and AMP-Activated Protein Kinase in Cell Growth Control

Sravanth K. Hindupur, Asier González and Michael N. Hall

Small but Mighty: Cell Size and Bacteria

Petra Anne Levin and Esther R. Angert
Mechanical Forces and Growth in Animal Tissues Loïc LeGoff and Thomas Lecuit

Biological Scaling Problems and Solutions in

Amphibians

Daniel L. Levy and Rebecca Heald

Intracellular Scaling Mechanisms Simone Reber and Nathan W. Goehring

Growing an Embryo from a Single Cell: A Hurdle in Animal Life Patrick H. O'Farrell

Organ-Size Regulation in Mammals Alfredo I. Penzo-Méndez and Ben Z. Stanger

Size Control in Plants--Lessons from Leaves and Flowers

Hjördis Czesnick and Michael Lenhard

Nuclear DNA Content Varies with Cell Size across

Human Cell Types

James F. Gillooly, Andrew Hein and Rachel

Damiani

Subcellular Size

Wallace F. Marshall

For additional articles in this collection, see http://cshperspectives.cshlp.org/cgi/collection/

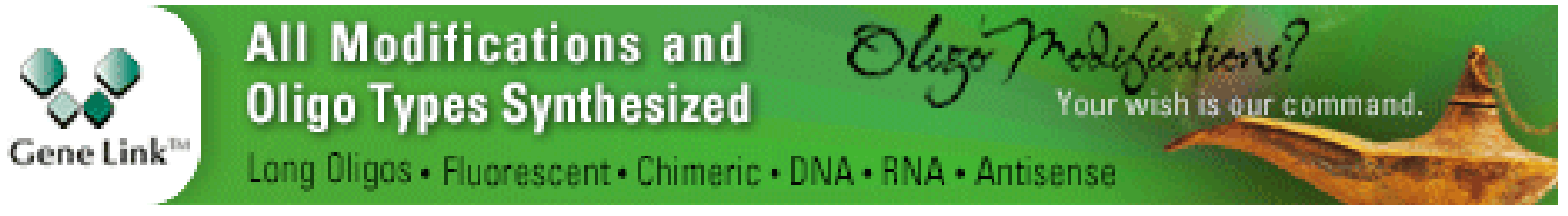

Copyright @ 2015 Cold Spring Harbor Laboratory Press; all rights reserved 